\title{
GLASS TUBE CLEAVING WITH PHASE-CORRECTED NON-DIFFRACTING LASER PULSES
}

\author{
ICALEO 2021, Talk MICRO 504, Paper \#0548_0779_000061
}

\author{
Daniel Flamm ${ }^{1,2}$, Jonas Kleiner ${ }^{1}$, Myriam Kaiser ${ }^{1}$, Felix Zimmerman ${ }^{1}$, and Tim Hesse ${ }^{1}$ \\ ${ }^{1}$ TRUMPF Laser- und Systemtechnik GmbH, Johann-Maus-Str. 2, 71254 Ditzingen \\ ${ }^{2}$ Corresponding author: daniel.flamm@trumpf.com
}

\begin{abstract}
Ultrashort laser pulses are used to modify complex inner and outer contours from glass tubes. The processing optics is designed to shape non-diffracting beams and to apply a sensitive phase correction for the processing behind curved interfaces. This enables single-pass, fullthickness modifications with feed rates in the order of $100 \mathrm{~mm} / \mathrm{s}$ are demonstrated. Final article separation is performed by thermal stress or via chemical etching.
\end{abstract}

\section{Introduction}

For the long-term storage of vital liquids, there is almost no alternative to materials made of glasses or glass ceramics [1]. Glass articles for clinical use, such as viols, ampoules or syringes, are consumed in large quantities, as they exhibit remarkable chemical, thermal and mechanical properties [1]. A common feature of these articles is a curved surface, which locally often exhibits cylindrical symmetry. Conventional laser machining strategies such as the well-established single-pass cutting using non-diffracting beams cannot be applied without further ado, as the curved interface and refractive index mismatch yield aberrated laser foci [2].

We will present methods and processing optics that allow for single-pass, full-thickness material modifications of glass tubes using ultrashort laser pulses. Sensitive phase correction is required to precompensate for occurring aberrations and to generate adapted non-diffracting beams retaining their salient features even for strong curvatures (local radii $<5 \mathrm{~mm}$ ) or large wall thicknesses $(>1 \mathrm{~mm})[2,3]$. A successful energy deposition inside the volume of the transparent tubes is confirmed by elongated type-III-regime modifications [4] enabling the separation of outer contours through the application of mechanical or thermal stress. The latter is particularly sought for transparent materials with low expansion coefficients, such as for borosilicate glasses. By exploiting $20 \mathrm{~W}$ average power from a TruMicro Series 2000 laser maximum feed rates of $>100 \mathrm{~mm} / \mathrm{s}$ were achieved [2]
The glass articles fabricated this way meet the highquality criteria of the pharmaceutical and healthcare industry with regard to surface quality and micro-debris.

\section{Non-diffracting Beams Behind Cylindrically Curved Interfaces}

Among many others, one of the most salient properties of non-diffracting beams is that they can be focused to any depth behind a plane interface, cf. Fig. 1 (a). The class of non-diffracting beams could therefore be successfully used for glass cleaving experiments with substrate thicknesses above $10 \mathrm{~mm}[5,6]$.

The situation of a non-diffracting propagating through a curved interface is less robust. A glass tube characterized by a cylindrical interface of radius $R_{T}$ represents the simplest form of a curved glass surface. As can be seen in the schematic of Fig. 1 (b), this interface acts as cylindrical lens resulting in an aberrated non-diffracting beam [the cases depicted in Fig. 1 (a) and (b) illustrate the propagation behaviour of a Bessel-Gaussian beam]. Depending on the glass tube geometry (refractive index $n_{2}$, radius of curvature $R_{T}$ and wall thickness $w_{T}$ ) the ideal on-axis constructive interference is disturbed in a way that strong intensity modulations appear. Resulting heterogeneous material modifications will prevent obtaining high quality glass edges or will, basically, prevent the separation along a desired contour [2].

To pre-compensate the induced aberrations, we assume that the cylindrical glass interface acts ideal thin phase element with quadratic phase modulation [2]

$$
\Phi_{c y l}^{\prime}(x)=\pi x^{2} / \lambda f_{c y l}^{\prime}
$$

with the lens' focal length $f_{c y l}^{\prime} \approx R_{T} / \Delta n$. Optical access to the $z$-position of the curved surface is provided virtually using a telescopic setup with given magnification $M=f_{2} / f_{1}$ [2]. If the start (z-position) of the non-diffracting beam is close to the curved optical interface, the telescopic setup allows to perform the beam shaping and the aberration correction within a single element, see axicon-like element depicted in 


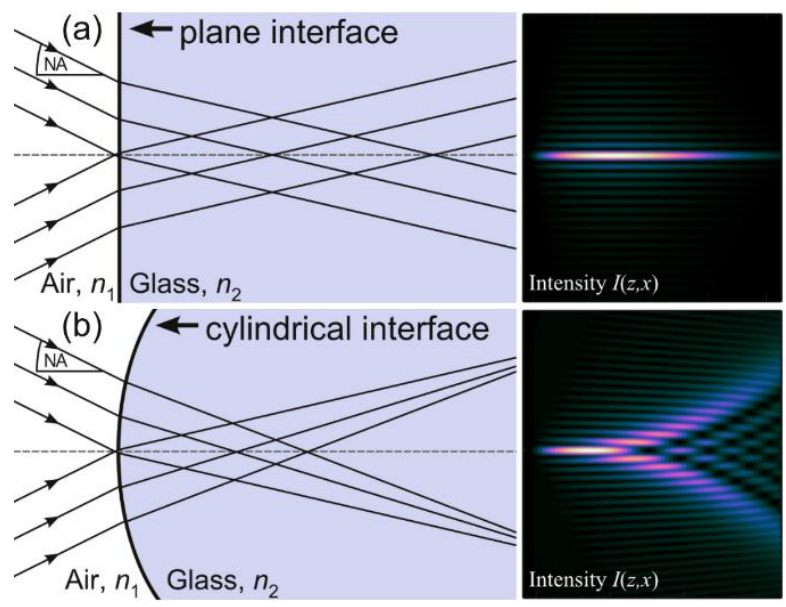

Figure 1. Ray and wave optical representation of the focus situation of a Bessel-Gaussian beam behind a plane (a) and a cylindrically curved (b) glass interface [2]

Fig. 2 (a). In the present case, the magnified phase aberration compensation $\Phi_{c y l} \approx-M^{2} \Phi_{c y l}^{\prime}$ may be realized as refractive element with a cylindrical surface of radius $R_{c y l} \approx-M^{2} R_{\mathrm{T}}$. Equally conceivable is the diffractive realization as single element with multiplexed phase modulations of an axicon $\Phi_{a x i}$ and the described cylindrical lens $\Phi_{c y l}$. We prefer the latter variant because it eliminates the need for laborious adjustment of the two components (axicon with respect to cylindrical lens). Thus, the generation of the nondiffracting beam and its aberration correction is performed by a single central beam shaping element. For the experiments conducted in this work the phase of the raw beam was modulated using a flexible liquidcrystal-on-silicon based spatial light modulator (SLM) [2].

The efficacy of our beam shaping concept is demonstrated by means of Fig. 3. Here, measured intensity profiles $I(x, z)$ are depicted for three different cases [2].

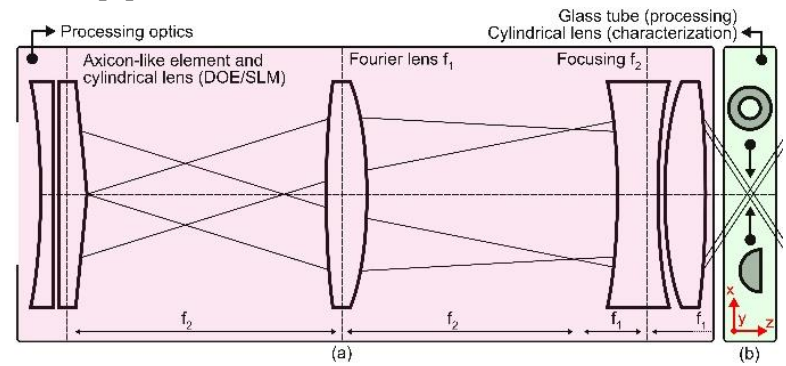

Figure 2. Optical realisation for shaping aberration-corrected non-diffracting beams (a). Beam shaping and phase-correction can be achieved using several components (axicon and cylindrical lenses) or single elements with multiple optical functionalities (DOEs, SLMs). The setup allows to flexibly switch between glass tubes and cylindrical lenses (b) in the focus of the non-diffracting for processing and adjustment/laser beam characterization, respectively $[2,6]$.
First, the propagation behavor of the Bessel- Gaussian beam behin a plane interface is shown (a). The second case (b) depicts the aberrated situation confirming our theory, cf. Fig. 1 (b). Finally, in the third row (c), the measured caustics exhibits almost identical focus properties as in the desired ideal case. High intensities, confined to the optical axis $(x=0)$ were measured at a material thickness of $z>1.5 \mathrm{~mm}$. We, thus, expect successful laser modifications along the entire wall thickness of our glass tubes with $w_{T} \sim 1 \mathrm{~mm}$.

\section{Single-pass, Full-thickness Modifications of Glass Tubes}

In the following, material processing experiments are conducted using the processing optics shown in Fig. 2 (a). Ultrashort laser pulses are illuminating the axiconlike element shaping the aberration-corrected nondiffracting beam. The optical axis of the processing optics is aligned perpendicular to the glass tube's tangential surface. During the laser modification step, tubes are rotated around their symmetry axis enabling feed rates of $>100 \mathrm{~m} / \mathrm{s}$ by employing $20 \mathrm{~W}$ of average power from a TruMicro 2000 laser system, see Fig. 4 (a), (b). Pulse energy, burst parameters and spatial pulse distances are adequately chosen to (mainly) generate elongated type-III-regime modifications [4] along the entire wall thickness of borosilicate tubes with a single pass. The entirety of modifications acts as breaking layer at which the actual separation is achieved. Fullthickness modifications are confirmed by investigating

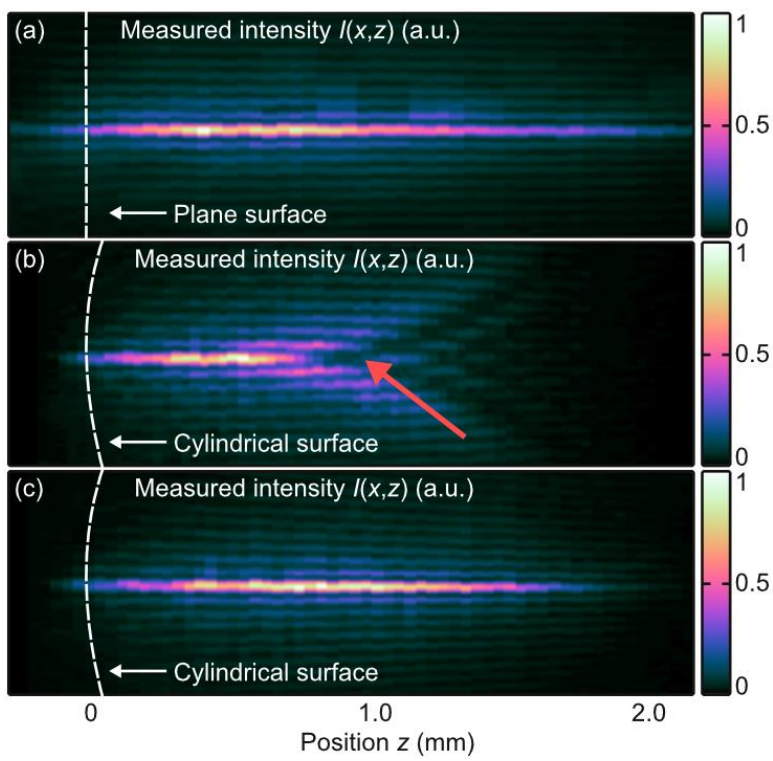

Figure 3. Measured intensity cross sections of non-diffracting beams propagating behind a plane interface (a), a cylindrically curved interface without aberration correction (b) and with applied aberration correction (third row) [2] Please note, the excellent agreement of distinct intensity features such as onaxis intensity modulations [red arrows in (b)]. 
the modification quality of the outer contour's edge. In Fig. 4 (c), (d) microscope images of details of tube edges are depicted that were separated mechanically. Elongated modifications ranging from the curved entrance surface to approximately half the wall thickness can be seen for the case of processing with an aberrated non-diffracting beam. In contrast, the processing with aberration corrected non-diffracting beams enable the formation of uniform modifications along the entire thickness, as shown in Fig. 4 (d).
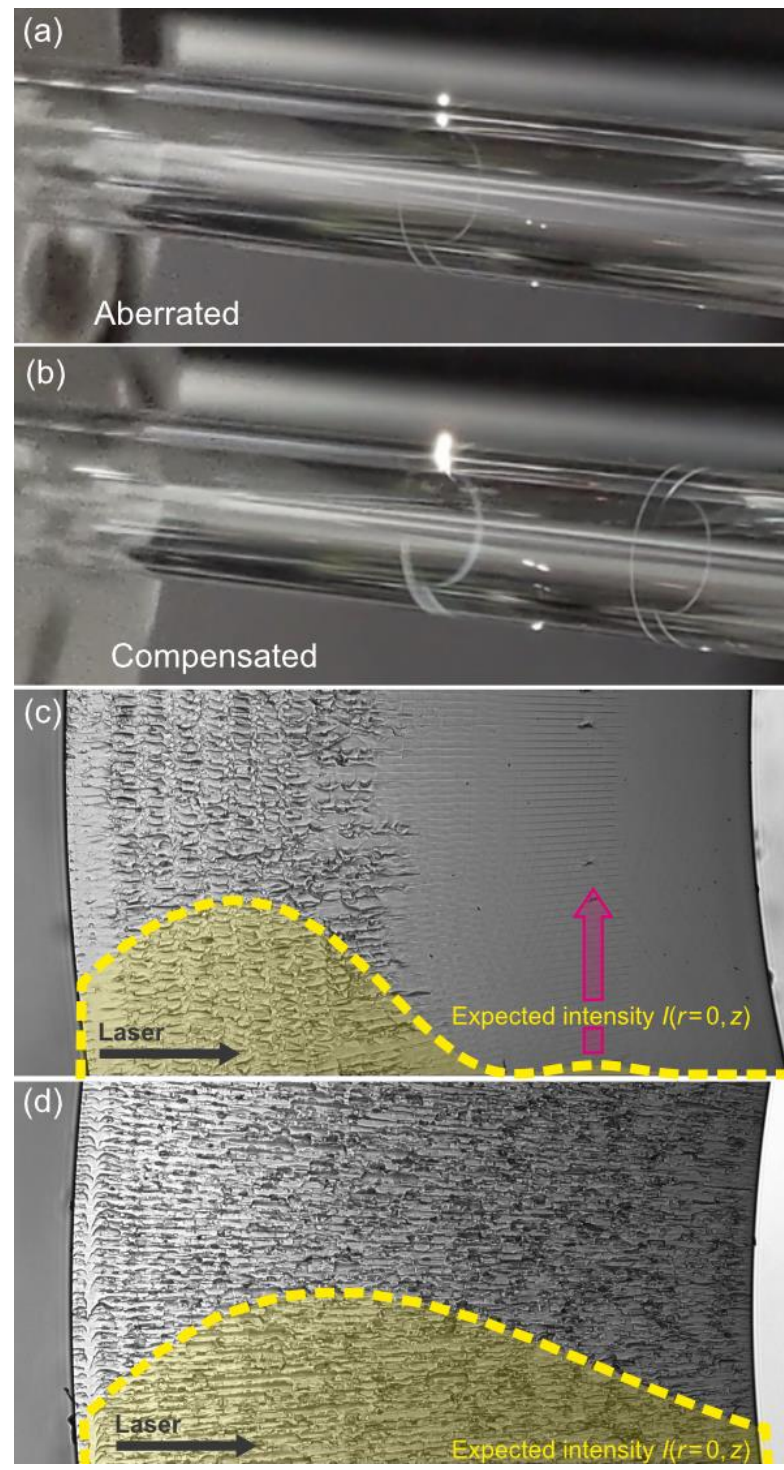

Figure 4. Camera recordings of the laser modification process and microscope images of borosilicate tube edges after mechanical separation. Plasma response of the glass tube during processing without (a) and with (b) applied phase correction. Elongated type-III-regime modifications that extend from entrance to approx. half the wall thickness (c). Processing with aberration-corrected non-diffracting beams (d) with uniform modifications along the entire wall thickness [2].
Although despite insufficiently long modifications, mechanical separation was successful in the first case [Fig. 4 (c)], the required breaking force was increased by several factors compared to the case with fullthickness modifications [Fig. 4 (d)]. Additionally, this increases the probability of generating chippings and a higher level of micro debris during the separation process [2].

\section{Processing Results}

Due to its relevance for the medical and pharmaceutical industry we focus on tubes made of borosilicate glass characterized by radii of $R_{T} \sim 4 \mathrm{~mm}$ and wall thicknesses of $w_{T} \sim 1 \mathrm{~mm}$. We would like to emphasize at this point that used laser parameters are strongly dependent on the respective glass type and tube geometry.

Figure 5 depicts the edge of a separated glass tube which was processed from $\sim 200 \mu \mathrm{J}$ pulses emerged from a TruMicro 2000 Series laser. Using $20 \mathrm{~W}$ of average power feed rates of $>100 \mathrm{~mm} / \mathrm{s}$ were achieved. The tube edge's measured mean surface roughness was measured to $S_{a}<1 \mu \mathrm{m}$ using a laser scanning microscope. The required flexural load for mechanical separation based on a four-point bending test was $\sim 50 \mathrm{~N}$. This comparatively low value, which was achieved in particular through the use of an asymmetric nondiffracting beam and crack control $[6,7]$ enables a thermally induced mechanical stress-based separation for borosilicate tubes.

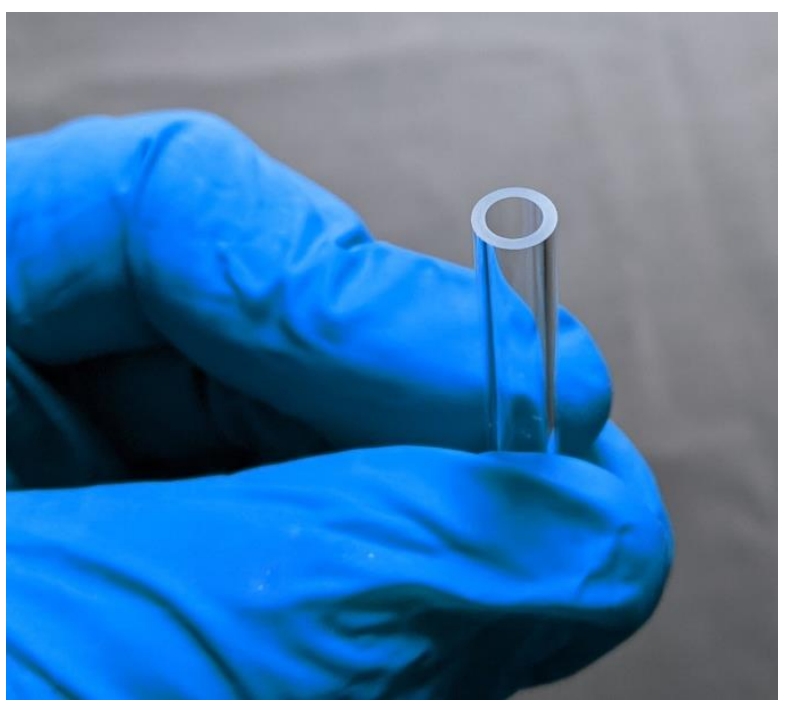

Figure 5. Mechanically separated glass tube made of borosilicate glass. Volume modifications were achieved using an adapted TOP Cleave cutting optics and a TruMicro Series 2000 laser in a single pass. Mean surface roughness of the tube edge was determined to $S_{a}<1 \mu \mathrm{m}$ (area evaluation). By exploiting $20 \mathrm{~W}$ of average power, feed rates of $>100 \mathrm{~mm} / \mathrm{s}$ were achieved [2]. 


\section{Conclusion}

Ultrashort pulsed laser cutting of glass tubes was demonstrated and applied to borosilicate samples exhibiting radii of $R_{T}>1 \mathrm{~mm}$. The developed processing optics generates an aberration corrected non-diffracting beam which enables single-pass, full-thickness modifications with feed rates $>100 \mathrm{~mm} / \mathrm{s}$ using $20 \mathrm{~W}$ of average power from a TruMicro 2000 Series laser. Achieved edge qualities with mean surface roughness parameters of $S_{a}<1 \mu \mathrm{m}$ meet the demands of the medical industry regarding chipping and micro debris. Our concept enables a thermal separation strategy even for borosilicate samples proven by minor loads required for mechanical separation with flexural bend tests [2].

\section{References}

[1] El-Meliegy, Emad, and Richard Van Noort. Glasses and glass ceramics for medical applications. Springer science $\&$ business media, 2011.

[2] Rave, Henning, et al. "Glass tube cutting with aberration-corrected non-diffracting ultrashort laser pulses." Optical Engineering 60.6 (2021): 065105 .

[3] Rave, Henning, et al. "Glass tube cutting for medical applications using ultrashort-pulsed lasers." Frontiers in Ultrafast Optics: Biomedical, Scientific, and Industrial Applications XXI. Vol. 11676. International Society for Optics and Photonics, 2021.

[4] Itoh, Kazuyoshi, et al. "Ultrafast processes for bulk modification of transparent materials." MRS bulletin 31.8 (2006): 620-625.

[5] Flamm, Daniel, et al. "Structured light for ultrafast laser micro- and nanoprocessing." Optical Engineering 60.2 (2021): 025105 .

[6] Jenne, M., et al. "Multi pulse pump-probe diagnostics for development of advanced transparent materials processing." Journal of Laser Micro Nanoengineering 13.3 (2018): 273279.

[6] Jenne, Michael, et al. "Facilitated glass separation by asymmetric Bessel-like beams." Optics express 28.5 (2020): 6552-6564.
[7] Chen, Keyou, et al. "Generalized axicon-based generation of nondiffracting beams." arXiv preprint arXiv:1911.03103 (2019). 